\title{
CAS-Complications Rate and Relation to Risk Factors*
}

\author{
Kamil Zeleňák ${ }^{1 \#}$, Vladimír Nosál ${ }^{2 \#}$, Jana Zeleňáková ${ }^{2}$ Egon Kurča ${ }^{2}$, \\ Igor Šinák ${ }^{3}$, Štefan Sivák ${ }^{2}$, Hubert Poláček ${ }^{1}$ \\ ${ }^{1}$ Department of Radiology, University Hospital, Martin, Slovakia \\ ${ }^{2}$ Department of Neurology, University Hospital, Martin, Slovakia \\ ${ }^{3}$ Department of Surgery, University Hospital, Martin, Slovakia \\ Email: " zelenak@unm.sk, ${ }^{\#}$ Vladimir.Nosal@jfmed.uniba.sk
}

Received April 3, 2012; revised December 10, 2012; accepted December 18, 2012

\begin{abstract}
Purpose: The aim of the study was to evaluate the risk factors for CAS with cerebral protection and the incidence of complication in patients with severe carotid stenosis. Materials and Methods: Overall, 95 consecutive patients with an average age of 64.67 years $(28$ - 83) (16 women $(17 \%)$ and 79 men $(83 \%))$ were enrolled in the prospective monocentric study. Indication for CAS was symptomatic carotid stenosis $\geq 70 \%(\mathrm{n}=60(63.16 \%))$ or asymptomatic stenosis $\geq 80 \%(\mathrm{n}=35(36.84 \%))$ according to the NASCET criteria on DSA, which were not suitable for surgery. Results: From the selected risk factors, the most frequently found were arterial hypertension in 87 patients $(91.6 \%)$, ischemic heart disease in $70(73.7 \%)$, hyperlipidemia in $61(64.2 \%)$, history of stroke in $43(45.3 \%)$, diabetes mellitus in 35 (36.8\%), smoking in $13(13.7 \%)$, and age more than 75 years in $9(9.5 \%)$. Significantly more frequent complications in elderly patients (more than the age of 75 years) were experienced: TIA $(p=0.049)$, early death $(p=0.049)$, restenosis $(p=0.04)$, and overall mortality $(p=0.04)$. Conclusion: Among the studied risk factors, only patients more than the age of 75 years were affected by the incidence of serious complications.
\end{abstract}

Keywords: CAS; Carotid Artery Stenting; Risk Factors; Elderly Patients; Age

\section{Introduction}

Stroke is the third most common cause of death in industrialized countries and is the leading cause of disability of patients [1]. Approximately $75 \%$ of all strokes are ischemic strokes, the remaining are hemorrhagic stroke, subarachnoid hemorrhage, and other much rarer clinical entities. Stenosis, or even occlusion of the internal carotid artery diameter, is a significant risk factor for ischemic stroke. Stenosis is most commonly caused by atherosclerosis. The correct choice of therapeutic intervention is a very important element of the management of patients with atherosclerotic carotid disabilities. In addition to general measures in terms of modifications of influenceable risk factors, within the therapeutic procedures are pharmacological treatment and invasive procedures such as carotid endarterectomy and CAS. Detailed knowledge of the risks, complications, and benefits of these procedures will allow for the optimal selection of patients for any given type of treatment.

Carotid artery stenting (CAS) with cerebral protection is one of the therapeutic treatment options of hemodynamically significant stenosis of the internal carotid artery. The aim of the study was to evaluate the

\footnotetext{
${ }^{*}$ The authors declare that they have no conflict of interest.

${ }^{\#}$ Corresponding authors.
}

risk factors for CAS with cerebral protection and the incidence of complications in patients with severe carotid stenosis.

\section{Materials and Methods}

The incidence of early and late complications, the occurrence of selected risk factors, and their possible impact on the incidence of serious complications during CAS with cerebral protection were evaluated in a prospective monocentric clinical study.

\subsection{Patient Selection}

The study included all patients who during the period of 2001 to 2008 had undergone implantation of a carotid stent with cerebral protection. The degree of stenosis was estimated by ultrasound and subsequent confirmation scan (CT angiography, contrast-enhanced MR angiography, or digital subtraction angiography (DSA)). The degree of carotid stenosis on the angiography was crucial for the CAS indication.

CAS was indicated by the consensus of the neurologist, interventional radiologist, and vascular surgeon.

Indication for CAS was symptomatic (TIA or stroke in the region supplied by stenotic artery) carotid stenosis 
$\geq 70 \%(\mathrm{n}=60(63.16 \%))$ or asymptomatic stenosis $\geq 80 \%$ ( $\mathrm{n}=35(36.84 \%))$ according to the NASCET criteria on DSA, which were not suitable for surgery due to: 1) Anatomical criteria: lesion at C-2 or higher, tandem stenosis, prior ipsilateral CEA, prior radical neck surgery or radiation, contralateral carotid occlusion, incomplete circle of Willis, non-atherosclerotic stenotic lesion (e.g. carotid dissection), contralateral laryngeal nerve palsy, short obese neck, cervical spine immobility, tracheostoma; 2) Comorbidities: severe cardiac and pulmonary disease.

\subsection{Clinical Protocol}

From the patient's medical history and records, we were particularly interested in the following data: patient's age, sex, history of stroke, smoking, hyperlipidemia, ischemic heart disease, arterial hypertension, diabetes mellitus, and previous revascularization intervention (carotid endarterectomy, carotid stent implantation). The patients were clinically examined by a neurologist, and the carotid artery was examined by an ultrasound both before and after CAS and at intervals of every 1 month, 3 months, and 6 months. CT (Computed tomography) or MR (magnetic resonance) of the brain was performed both before and after CAS. The incidence of early and late complications was monitored.

\subsection{Definitions}

Periprocedural complications (up to 24 hours after the procedure) or those that occurred within 30 days after intervention were considered early complications. Late complications were complications that occurred after 30 days of intervention. A complication was defined as an event according to the rules of clinical practice. A major stroke was defined as a new neurological deficit that persisted at 30 days and that changed the National Institutes of Health Stroke Scale by $\geq 4$. A minor stroke was defined as a new neurological deficit that was either resolved completely within 30 days or increased the National Institutes of Health Stroke Scale by $\leq 3$. Transient ischemic attack (TIA) was defined as a brief episode of neurological dysfunction caused by focal brain or retinal ischemia, with clinical symptoms typically lasting less than one hour, and without evidence of acute infarction by radiological techniques. Serious complications of CAS are death, formation of focal neurological deficit, and significant restenosis. Nonserious complications are those that do not pose a serious risk to the patient according to GCP (good clinical practice).

\subsection{Medication}

All patients received dual-antiplatelet pharmacological pre-treatment. At least 3 days before carotid artery stenting, the patients received acetyl salicylic acid (ASA) at a dose of $100 \mathrm{mg}$ daily, and clopidogrel at a dose of 75 $\mathrm{mg}$ daily or ticlopidine at a dose of $250 \mathrm{mg}$ twice daily. During the procedure and the next 24 hours during which the patients were heparinized, APTT (Activated partial thromboplastine time) was 2 to 3 times longer. After the procedure, the patients received $75 \mathrm{mg}$ of clopidogrel or $2 \times 250 \mathrm{mg}$ of ticlopidine daily for 30 days and $100 \mathrm{mg}$ of ASA daily for at least 6 months.

\subsection{Technique}

All interventions were performed by one interventional radiologist. The procedure was performed under local anesthesia according to the standardized protocol, and the transfemoral approach was used in all the patients. The type of stent and filter was chosen according to the type of stenosis and vessel tortuosity by an interventional radiologist. Contralateral internal carotid artery occlusion was not a contraindication for the procedure.

\subsection{Statistical Analysis}

The obtained data were statistically processed by using the statistical program, SPSS version 14.0. When processing the data, we used frequency analysis and bivariate correlation analysis.

\section{Results}

The total number of patients enrolled in the study was 95 (16 women $(17 \%)$ and 79 men $(83 \%))$. The average age was $64.67(28-83)$ years. The number of patients older than or equal to 75 years was $9(9.5 \%)$.

The occurrence of selected risk factors is shown in Table 1. We discovered that the most frequent risk factors are arterial hypertension in 87 patients, coronary heart disease in 70 patients, and hyperlipidemia in 61 patients. Furthermore, the medical history showed that 43 patients had history of stroke, 35 patients had diabetes mellitus, and 13 patients were smokers.

Table 1. Percentage of occurrence of selected risk factors.

\begin{tabular}{ccc}
\hline Risk factor & Number of patients $(\mathrm{n}=95)$ & Percentage \\
\hline Hypertension & 87 & $91.6 \%$ \\
Ischemic heart disease & 70 & $73.7 \%$ \\
Hyperlipidemia & 61 & $64.2 \%$ \\
History of stroke & 43 & $45.3 \%$ \\
Diabetes mellitus & 35 & $36.8 \%$ \\
Smoking & 13 & $13.7 \%$ \\
Age $\geq 75$ years & 9 & $9.5 \%$ \\
\hline
\end{tabular}




\subsection{Early Complications}

Periprocedural complications (up to 24 hours after the procedure) were as follows: $1 \times$ TIA (1.05\%), $2 \times$ embolization to the ophtalmic artery (rated as minor stroke) $(2.1 \%)$, and $1 \times$ major stroke $(1.05 \%)$. From the serious early complications (within 30 days, including periprocedural complications), TIA occurred in 2 patients $(2.1 \%)$, stroke in 4 patients $(4.2 \%)(3 \times$ minor strokes, $1 \times$ major stroke), and death in $2(2.1 \%)$ patients ( 1 for ischemic stroke 12 days later and 1 for malignant cardiac arrhythmias 6 days after intervention) (Table 2). Bradycardia occurred in 12 patients $(12.7 \%)$, hypotension occurred in 30 patients $(31.6 \%)$, hypotension combined with bradycardia occurred in 31 patients $(32.6 \%)$, and serious bradycardia and hypotension occurred in one patient $(1.1 \%)$, requiring urgent administration of medi- cation. Febrile state was observed in 3 patients $(3.2 \%)$, hemorrhage from the puncture site (requiring blood transfusion in a patient with primary anemia) without the usage of a closure device and without the necessity of surgery in 2 patients $(2.1 \%)$, allergic reaction (urticaria) to the contrast media after the procedure in 1 patient $(1.1 \%)$, urinary tract infection in 2 patients $(2.1 \%)$, local hematoma at the puncture site without the necessity of surgery in 3 patients (3.2\%), asymptomatic occlusion of external carotid artery in 1 patient $(1.1 \%)$, iliac artery dissection in 1 patient $(1.1 \%)$, artery pseudoaneurysm in 1 patient $(1.1 \%)$, bronchopneumonia in 1 patient $(1.1 \%)$, occlusion of the filter in 1 patient $(1.1 \%)$, and femoral artery occlusion successfully treated by endovascular technique in 1 patient $(1.1 \%)$. The number and percentage of complications are mentioned in Table 3.

The incidence of serious early death or stroke (without

Table 2. Clinical outcomes.

\begin{tabular}{ccc}
\hline $\mathrm{n}=95$ & Periprocedural complication (up to 24 hours) & Early complication (within 30 days, including periprocedural) \\
\hline Death & $0(0 \%)$ & $2(2.1 \%)$ \\
Major stroke & $1(1.05 \%)$ & $1(1.05 \%)$ \\
$\begin{array}{c}\text { Minor stroke } \\
\text { (including retinal embolization) }\end{array}$ & $2(2.1 \%)$ & $3(3.16 \%)$ \\
TIA & $1(1.05 \%)$ & $2(2.1 \%)$ \\
\hline
\end{tabular}

Table 3. Incidence of early complications.

\begin{tabular}{|c|c|c|}
\hline Complication & Number $(\mathrm{n}=95)$ & Percentage \\
\hline Death & 2 & $2.1 \%$ \\
\hline Stroke $(3 \times$ minor and $1 \times$ major $)$ (including retinal embolization) & 4 & $4.2 \%$ \\
\hline TIA & 2 & $2.1 \%$ \\
\hline Bradycardia & 12 & $12.7 \%$ \\
\hline Hypotension & 29 & $31.6 \%$ \\
\hline Bradycardia and hypotension & 31 & $32.6 \%$ \\
\hline Febrile state & 3 & $3.2 \%$ \\
\hline Hemorrhage from the puncture site & 2 & $2.1 \%$ \\
\hline Allergy to contrast media (urticaria) after CAS & 1 & $1.1 \%$ \\
\hline Urinary tract infection & 2 & $2.1 \%$ \\
\hline Local hematoma in the puncture site without necessity of surgery & 3 & $3.2 \%$ \\
\hline Local infection at the puncture site & 1 & $1.1 \%$ \\
\hline Asymptomatic occlusion of the external carotid artery & 1 & $1.1 \%$ \\
\hline Iliac artery dissection & 1 & $1.1 \%$ \\
\hline Pseudoaneurysm & 1 & $1.1 \%$ \\
\hline Bronchopneumonia & 1 & $1.1 \%$ \\
\hline Occlusion of the filter & 2 & $2.1 \%$ \\
\hline Femoral artery occlusion treated by endovascular technique & 1 & $1.1 \%$ \\
\hline
\end{tabular}


retinal embolization) was observed in a total of 4 cases $(4.2 \%)$.

From the late complications, asymptomatic restenosis occurred in $6(6.3 \%)$ patients, and death occurred in 4 (4.2\%) patients (Table 4).

By analyzing the causes of death, we found that in the 30-day interval, there were two early deaths. One patient (male, 53 years) died 12 days after intervention due to ischemic stroke, and one patient (male, 83 years) died because of malignant cardiac arrhythmias 6 days after intervention. The average number of days from intervention to death was 9 .

Four patients died in the interval of more than 30 days after intervention. The causes of death were as follows: 1) A 77-year-old man died of a brain tumor 540 days after intervention; 2) A 78-year-old man died because of a myocardial infarction 618 days after intervention; 3) A 69-year-old man died due to cardiac failure 1157 days after intervention; and 4) A 56-year-old woman died of meningitis 35 days after intervention. We note that the average number of days from intervention to death was 587.5 .

Overall, the incidence of death after intervention without a time limit was observed in 6 patients $(6.3 \%)$ on an average of 394.4 days from the date of intervention.

\subsection{Analysis of the Impact of Risk Factors on the Incidence of Serious Early and Late Complications}

Higher age played a role in the incidence of early serious complications such as death and TIA. We found that one of the total of 2 deaths within 30 days after intervention occurred in elderly patients, which represents a marginally significant impact on the incidence of this complication $(p=0.049)$. None of the patients surpassed stroke in the early period after intervention. One elderly patient had TIA (from a total of two), which represents a marginally significant impact on the incidence of specified complications $(\mathrm{p}=0.049)$. Late complications were death and restenosis. Higher age did not have a significant impact on the incidence of death $(p=0.284)$, because only one elderly patient died. However, we point out that higher age significantly influenced the incidence of restenosis $(p=0.040)$. From the total of six restenoses, two occurred in elderly patients. Overall, in the studied file, six deaths were recorded, in which 2 patients were

Table 4. The incidence of late complications.

\begin{tabular}{ccc}
\hline Type of complication & Number $(\mathrm{n}=95)$ & Percentage \\
\hline Asymptomatic restenosis & 6 & $6.3 \%$ \\
Death (no fatal stroke) & 4 & $4.2 \%$ \\
\hline
\end{tabular}

elderly. The result of correlation analysis was that higher age has a significant impact on overall mortality $(\mathrm{p}=$ 0.040).

From serious early complications, there were two early deaths in the group of patients who had stroke in their history $(\mathrm{p}=0.118)$, three patients had another stroke $(\mathrm{p}=$ $0.226)$, and none of the patients had TIA $(p=0.198)$. Late complications such as restenosis were recorded twice $(\mathrm{p}=0.549)$. In addition, after 30 days, we recorded one case of death $(\mathrm{p}=0.411)$. Overall, 3 patients die $(\mathrm{p}=$ 0.812 ) in this group.

Serious early complications that occurred in the group of patients with hypertension were as follows: two patients died $(\mathrm{p}=0.669)$, three patients had a stroke $(\mathrm{p}=$ $0.227)$, and two patients experienced a TIA $(\mathrm{p}=0.669)$. In terms of late complications in the group of hypertensive patients, we noticed that restenosis occurred 5 times $(\mathrm{p}=0.458)$, and death occurred thrice after 30 days $(\mathrm{p}=0.227)$. The total mortality in this group (patients with arterial hypertension) was five patients, which does not pose a significant impact of this risk factor $(\mathrm{p}=$ 0.458).

In the group of patients with ischemic heart disease, two early deaths occurred $(p=0.398)$, four patients had a stroke $(\mathrm{p}=0.226)$, and two patients had a TIA ( $\mathrm{p}=$ 0.398). We can observe that late complications such as restenosis occurred twice $(\mathrm{p}=0.549)$, and death also occurred twice after 30 days $(p=0.277)$. In terms of mortality, in this studied group, death was recorded in 4 patients $(\mathrm{p}=0.691)$.

In diabetic patients, we observed the following early serious complications: one early death $(p=0.7)$, two patients experienced a stroke $(\mathrm{p}=0.582)$, and none of the patients surpassed TIA $(\mathrm{p}=0.28)$. Late complications such as restenosis occurred in one patient $(p=0.295)$, and death occurred after 30 days in 2 patients $(\mathrm{p}=0.582)$. We can conclude that overall mortality (early and late deaths) involved three patients $(p=0.495)$.

Smoking had no significant impact on any of the major early or late complications. In this group, there was no early death $(p=0.574)$, none of the patients surpassed a stroke $(p=0.421)$, and none surpassed TIA $(p=0.574)$. Late complications such as restenosis occurred in two patients $(\mathrm{p}=0.151)$, and death occurred after 30 days in one patient $(\mathrm{p}=0.506)$. The overall mortality was only 1 patient $(\mathrm{p}=0.828)$.

From serious early complications in patients with hyperlipidemia, one early death $(p=0.676)$ occurred, two patients experienced a stroke $(\mathrm{p}=0.55)$, and one patient underwent TIA $(\mathrm{p}=0.676)$. Restenosis occurred in three patients $(\mathrm{p}=0.458)$, and three patients died after 30 days $(p=0.65)$. Four patients died $(p=0.898)$ in total.

The results are presented in Tables 5 and 6 . 
Table 5. Correlation analysis—serious early complications and their relationship to risk factors.

\begin{tabular}{ccccccccc}
\hline & Age & History of Stroke & Hypertension & Ischemic heart disease Diabetes mellitus Smoking & Hyperlipidemia \\
\hline \multirow{2}{*}{ Stroke } & Pearson Correlation & -0.068 & 0.125 & -0.125 & 0.125 & 0.057 & -0.083 & -0.062 \\
& Sig. (2-tailed) & 0.514 & 0.226 & 0.227 & 0.226 & 0.582 & 0.421 & 0.550 \\
\multirow{2}{*}{ TIA } & Pearson Correlation & $0.203^{*}$ & -0.133 & 0.044 & 0.088 & -0.112 & -0.058 & -0.043 \\
& Sig. (2-tailed) & 0.049 & 0.198 & 0.669 & 0.398 & 0.280 & 0.574 & 0.676 \\
\multirow{2}{*}{$\begin{array}{l}\text { Early } \\
\text { death }\end{array}$} & Pearson Correlation & $0.203^{*}$ & 0.161 & 0.044 & 0.088 & 0.040 & -0.058 & -0.043 \\
\hline
\end{tabular}

${ }^{*}$ Significant $(\mathrm{p}<0.05)$.

Table 6. Correlation analysis-late complications and their relationship to risk factors.

\begin{tabular}{ccccccccc}
\hline & Age & History of Stroke Hypertension Ischemic heart disease Diabetes mellitus Smoking & Hyperlipidemia \\
\hline \multirow{2}{*}{ Restenosis } & Pearson Correlation & $0.212^{*}$ & -0.062 & -0.077 & -0.140 & -0.109 & 0.148 & -0.077 \\
& Sig. (2-tailed) & 0.040 & 0.549 & 0.458 & 0.177 & 0.295 & 0.151 & 0.458 \\
\multirow{2}{*}{$\begin{array}{c}\text { Death after } \\
\text { 30 days }\end{array}$} & Pearson Correlation & 0.111 & -0.085 & -0.125 & -0.113 & 0.057 & 0.069 & 0.047 \\
\multirow{2}{*}{$\begin{array}{c}\text { Overall } \\
\text { mortality }\end{array}$} & Sig. (2-tailed) & 0.284 & 0.411 & 0.227 & 0.277 & 0.582 & 0.506 & 0.650 \\
& Pearson Correlation & $0.212^{*}$ & 0.025 & -0.077 & -0.041 & 0.071 & 0.023 & 0.013 \\
\hline
\end{tabular}

*Significant $(\mathrm{p}<0.05)$.

\section{Discussion}

The primary objective of the implemented prospective clinical study was to identify and analyze commonly known and most frequent cardiovascular risk factors occurring in patients with hemodynamically significant stenosis of the internal artery treated by implantation of the carotid stent. Another goal was to monitor these patients for the incidence of any complications that arose during intervention or within a 30-day period after intervention (early complications) and also for serious late complications such as death and restenosis.

In our opinion, it is possible to consider that the results obtained are homogeneous, because all the interventions were performed by a single interventional radiologist, and the parameters required were monitored and evaluated in a single independent workplace. A disadvantage of this study is the relatively limited number of patients included.

An analysis of the relevant risk factors was performed in the presented study. As is apparent from the results, the group of patients with serious atherosclerotic disability of the internal carotid was significantly polymorbid. This is evidenced by the fact that the percentage of patients who develop three or more risk factors accounted for $81 \%$ of the sample. We found that the most frequent risk factor was arterial hypertension, which was present in nearly every patient ( $91.6 \%$ of patients), followed by coronary artery disease, hyperlipidemia, a positive history of ischemic stroke, diabetes mellitus, smoking, and high age. Additionally, the fact that only one patient did not have any of the risk factors present was remarkable. On the other hand, there were no patients in the study who had all seven of the selected risk factors present. Six risk factors were present in only one patient.

Based on the facts obtained, it can be concluded that from the perspective of the risk of ischemic stroke occurrence, almost all patients were in the high-risk groups, because of the presence of several serious risk factors and due to the incidence of hemodynamically significant internal carotid artery stenosis. This fact is supported by numerous studies in literature, which monitor these actual risk factors, but particularly, their relationship to the risk of ischemic stroke occurrence [2-4].

Overall, there was a relatively high incidence of nonserious complications. Complications, which we recorded in patients in this study, can be divided into several categories. From a time point of view: periprocedural, early, and late. From a clinical point of view: serious and non-serious. The most frequent complications were hypotension, bradycardia, and hypotension combined with bradycardia. Isolated hypotension occurred in $31.6 \%$ of all cases, and hypotension combined with bradycardia occurred in $32.6 \%$ of all cases. The number of the cases was the same in comparison with literature facts [5]. Although the complications did not ultimately affect the overall health of the patient, in some cases, intensive 
medication was required. All the cases were closely monitored. Some patients had to discontinue chronic antihypertensive medication to achieve normotension, and they have been released for home treatment without any antihypertensive treatment. It is not known how long the normotensive blood pressure values remained and how soon it was necessary to re-deploy anti-hypertensive treatment, because the blood pressure was not monitored on a long-term basis. The long-term monitoring of blood pressure could be an interesting subject for further clinical studies. The occurrence of hypotension and bradycardia has been explained as being due to the distension of sinus caroticus due to the implanted stent [6]. The results of our studies show that although the hypotension or bradycardia are not serious complications in patients with implanted carotid stent, it is necessary to keep in mind the possibility of this occurrence and complication, and to monitor the patient's vital function. The reason is that serious hypotension or bradycardia may cause cerebral hypoperfusion or contribute to the formation of acute hypoxemic stroke. In addition, one patient suddenly died within a short period after stent implantation due to cardiac arrhythmia. In this case, it is not definitely possible to exclude the impact of the stent to the emergence of the arrhythmia.

The serious complications, which we observed in the group of patients, include early death, the emergence of ischemic stroke, restenosis, and late death. In some respects, the emergence of transient cerebral hypoperfusion (TIA) can be regarded as a serious complication as well. However, the emergence of TIA is not considered a serious complication in larger clinical studies. On the other hand, there is a warning sign, which may ultimately result in a complete stroke, if ignored. The incidence of serious complications, which we found in our group of patients, can be regarded as either the same or lower as compared with these incidences in large clinical studies, including meta-analysis [7-13]. The number of early deaths and strokes was not high. Total long-term mortality within was six (6.3\%). Of this number, however, only one patient $(1.1 \%)$ died from cerebrovascular causes due to ischemic stroke, and three patients $(3.2 \%)$ died from cardiac causes. One patient developed meningitis with a lethal outcome 35 days after intervention, whereas the relationship with the intervention is particularly problematic. The last patient died from brain tumor, which cannot be linked to intervention.

In comparison with literature facts, it can be stated that our group of patients experienced a relatively low number of serious early and late complications. This can be justified by the fact that all the interventions were performed by a single experienced interventional radiologist, and, therefore, the possibility of errors by a less experienced interventionalist was reduced. It should also be noted that all of the restenoses of patients $(6.3 \%)$ were successfully treated by endovascular treatment. Additionally, it indicates that for this reason, the long-term postprocedural monitoring of patients is necessary.

In this prospective clinical study, we have focused our attention on the risk factors and their relationship to the occurrence of serious early and late complications. So far, a higher age was the single documented risk factor that had a significant impact on the incidence of serious complications. We rely on the criteria of the SPACE study [14], while dividing patients into the age groups (a group under 75 years and a group more than 75 years inclusive), primarily because of the better comparability and interpretation of results [11]. As in many published works $[7-12,15,16]$, higher age had, at the marginal significance level, an impact on the incidence of TIA and early death. Higher age also had a significant impact on the incidence of restenosis, and, therefore, understandably the group of elderly patients had a higher overall mortality rate. Bonati et al. state that CAS should be avoided in older patients [17]. We concluded that higher age is a relative contraindication to the intervention.

\section{Conclusion}

Patients enrolled in this prospective study were significantly polymorbid. The risk factors in the order of most frequent occurrence are arterial hypertension, ischemic heart disease, hyperlipidemia, stroke history, diabetes mellitus, smoking, and higher age. Insignificant early complications in the order of occurrence were most often hypotension, bradycardia, and hypotension combined with bradycardia. Serious early complications in the order of occurrence were most often stroke, TIA, and death. Among the late serious complications, restenosis and death occurred. All restenoses were clinically asymptomatic and were successfully treated by the endovascular technique. From the risk factors studied, higher age was the only factor that had a significant impact on the incidence of serious complications. We can confirm that our center utilized a safe CAS method of treatment, which provides a comparable incidence of early and late complications. Nevertheless, there is the need for larger clinical studies that verify the safety of this procedure. It is also necessary to search for other risk factors that may have a potential impact on the emergence of postprocedural complications.

\section{REFERENCES}

[1] World Health Organisation, "International Society of Hypertension Guidelines for the Management of Hypertension. Guidelines Subcommittee," Journal of Hypertension, Vol. 17, No. 2, 1999, pp. 151-183.

[2] A. Arboix, V. Cendrós, M. Besa, L. García-Eroles, M. 
Oliveres, C. Targa, M. Balcells, E. Comes and J. Massons, "Trends in Risk Factors, and Outcome Stroke Subtypes," Cerebrovascular Diseases, Vol. 26, No. 5, 2008, pp. 509516.

[3] R. L. Sacco, R. Adams, G. Albers, M. J. Alberts, O. Benavente, K. Furie, L. B. Goldstein, P. Gorelick, J. Halperin, R. Harbaugh, S. C. Johnston, I. Katzan, M. KellyHayes, E. J. Kenton, M. Marks, L. H. Schwamm, T. Tomsick, American Heart Association; American Stroke Association Council on Stroke, Council on Cardiovascular Radiology and Intervention and American Academy of Neurology, "Guidelines for Prevention of Stroke in Patients with Ischemic Stroke or Transient Ischemic Attack: A Statement for Healthcare Professionals from the American Heart Association/American Stroke Association Council on Stroke," Stroke, Vol. 37, No. 2, 2006, pp. 577617.

[4] R. L. Sacco, "Newer Risk Factors for Stroke," Neurology, Vol. 57, No. 5, 2001, pp. 31-34.

[5] R. Gupta, M. Horowitz and T. G. Jovin, "Hemodynamic Instability after Carotid Artery Angioplasty and Stent Placement: A Review of the Literature," Neurosurgical Focus, Vol. 18, No. 1, 2005, p. e6. doi:10.3171/foc.2005.18.1.7

[6] G. Pappadà, E. Beghi, R. Marina, E. Agostoni, C. Cesana, F. Legnani, M. Parolin, D. Petri and E. P. Sganzerla, "Hemodynamic Instability after Extracranial Carotid Stenting," Acta Neurochirurgica, Vol. 148, No. 6, 2006, pp. 639-645.

[7] E. Touzé, D. Calveta, G. Chatellierb and J. L. Mas, "Carotid Stenting," Current Opinion in Neurology, Vol. 21, No. 1, 2008, pp. 56-63. doi:10.1097/WCO.0b013e3282f313d2

[8] European Carotid Surgery Trialists Collaborative Group, "Randomized Trial of Endarterectomy for Recently Symptomatic Carotid Stenosis: Final Results of the MRC European Carotid Surgery Trial (ECST)," Lancet, Vol. 351, No. 9113, 1998, pp. 1379-1387.

[9] A. Halliday, A. Mansfield, J. Marro, C. Peto, R. Peto, J. Potter, D. Thomas and MRC Asymptomatic Carotid Surgery Trial (ACST) Collaborative Group, "Prevention of Disabling and Fatal Strokes by Successful Carotid Endarterectomy in Patients without Recent Neurological Symptoms: Randomised Controlled Trial," Lancet, Vol. 363, No. 9420, 2004, pp. 1491-1502.

[10] American College of Cardiology Foundation, American Society of Interventional \& Therapeutic Neuroradiology, Society for Cardiovascular Angiography and Interventions, Society for Vascular Medicine and Biology, Society of Interventional Radiology, E. R. Bates, J. D. Babb, D. E. Casey Jr., C. U. Cates, G. R. Duckwiler, T. E.
Feldman, W. A. Gray, K. Ouriel, E. D. Peterson, K. Rosenfield, J. H. Rundback, R. D. Safian, M. A. Sloan and C. J. White, "ACCF/SCAI/SVMB/SIR/ASITN 2007 Clinical Expert Consensus Document on Carotid Stenting: A Report of the American College of Cardiology Foundation Task Force on Clinical Expert Consensus Documents (ACCF/SCAI/SVMB/SIR/ASITN Clinical Expert Consensus Document on Committee Carotid Stenting," Journal of the American College of Cardiology, Vol. 49, No. 1, 2007, pp. 126-170.

[11] SPACE Collaborative Group, P. A. Ringleb, J. Allenberg, H. Brückmann, H. H. Eckstein, G. Fraedrich, M. Hartmann, M. Hennerici, O. Jansen, G. Klein, A. Kunze, P. Marx, K. Niederkorn, W. Schmiedt, L. Solymosi, R. Stingele, H. Zeumer and W. Hacke, "30 Day Results from the SPACE Trial of Stent-Protected Angioplasty versus Carotid Endarterectomy in Symptomatic Patients: A Randomized Noninferiority Trial," Lancet, Vol. 368, No. 9543, 2006, pp. 1239-1247.

[12] M. Bosiers, P. Peeters, K. Deloose, J. Verbist, H. Sievert, J. Sugita, F. Castriota and A. Cremonesi, "Does Carotid Artery Stenting Work on the Long Run: 5-Year Results in High-Volume Centers (Eloc Registry)," The Journal of Cardiovascular Surgery, Vol. 46, No. 3, 2005, pp. 241247.

[13] R. Stingele and P. A. Ringleb, "To Stent or Not to Stent: Stent-Protected Percutaneous Angioplasty versus Endarterectomy Post Hoc Analyzes," Current Opinion in Neurology, Vol. 22, No. 1, 2009, pp. 75-79.

[14] SPACE Collaborative Group, "30 Day Results from the SPACE Trial of Stent-Protected Angioplasty versus Carotid Endarterectomy in Symptomatic Patients: A Randomised Non-Inferiority Trial," Lancet, Vol. 368, No. 9543, 2006, pp. 1239-1247.

[15] R. Topakian, M. Sonnberger, K. Nussbaumer, H. P. Haring, J. Trenkler and F. T. Aichner, "Posprocedural HighDensity Lipoprotein Cholesterol Predicts Carotid Stent Patency at 1 Year," European Journal of Neurology, Vol. 15, No. 2, 2008, pp. 179-184.

[16] A. Mathur, G. S. Roubini, S. S. Iyer, C. Piamsonboon, M. W. Liu, C. R. Gomez, J. S. Yadav, H. D. Chastain, L. M. Fox, L. S. Dean and J. J. Vitek, "Predictors of Stroke Complicating Carotid Artery Stenting," Circulation, Vol. 97, No. 13, 1998, pp. 1239-1245.

[17] L. H. Bonati, G. Fraedrich and Carotid Stenting Trialists' Collaboration, "Age Modifies the Relative Risk of Stenting versus Endarterectomy for Symptomatic Carotidstenosis-A Pooled Analysis of EVA-3S, SPACE and ICSS," European Journal of Vascular and Endovascular Surgery, Vol. 41, No. 2, 2011, pp. 153-158. 